\title{
Colaborando a la bibliografía de San Agustín (1925-1945)
}

Cada día son más los exquisitos trabajos sobre san Agustín y se multiplican los estudios y las investigaciones minuciosas en torno a pequeños o grandes detalles en las más variadas ramas del saber, desde la breve nota filológica a la más concienzuda obra filosófica, doctrinal, genérica o teológica. Aunque parezca mentira y dado el inmenso caudal que arrastra la bibliografía, aún caben nuevos horizontes que otear. La bibliografía se acrecienta día a día y cuando se vuelve la vista atrás se percata uno de que hay mucho estudiado y a veces no recogido, pero siempre interesante. $Y$ en esos veinte años convulsos (1925-1945) del siglo XX se mezclaron las guerras con la inteligencia y surgieron filosofías en busca de fundamentos profundos y humanos, y en esa esfera de lo humano y sus valores trascendentes se hallaba en primera línea San Agustín.

Me ha parecido una traición a lo agustiniano no colaborar a este engrandecimiento de san Agustín con un aporte o colaboración de años a una bibliografía que ya se ha reunido en ocasiones, pero cuya puerta continúa todavía abierta. He recorrido muchas bibliotecas en diferentes hemisferios y países y he quemado años incontables en un trabajo denodado, quizá no valioso, pero tal vez capaz de encender alguna chispa más en el ya amplio campo agustiniano. No quisiera que por no ser completo, se perdiera parte de su valor y no viera la luz por miedos infundados. Yo sé que es muy dificil añadir algo nuevo a lo ya existente, pero también sé que si los medios de hoy son múltiples, un instrumento más, por escaso que sea o lo parezca, ayuda siempre a configurar la imagen de quien ha sido y continúa siendo mi pasión acrecida, un referente para la humanidad vivida y entendida.

Cuando consulté la posibilidad de la publicación de esta Colaboración a la Bibliografía de San Agustín (1925-1945), se me contestó que no había inconveniente, ya que el "Corpus Augustinianum Gessense" recoge 
unos 1800 títulos para ese período de tiempo y que quizá habría más sin duda alguna. Ni oposición ni competencia, solamente un aporte sin pretensiones, como un regalo a San Agustín con años de investigación y de estudio. Tal vez, al final, haya que decir que sea bienvenido todo aquel que coloque, aunque sea una piedrita más en ese hermoso e inmenso edificio del agustinismo.

Como en toda bibliografía habrá lagunas -todo es perfectible en este mundo-, pero trataré de dar una visión clara, sin crítica -es difícil enjuiciar tiempos pasados-, o simplemente dejaré al autor que hable por sí mismo y en su propia lengua. Todos aprenderemos algo y una vez más nos percataremos de que el siglo XX fue un siglo netamente agustiniano o al menos en el que muchos volvieron su vista a la vida y a las enseñanzas de San Agustín. Los veinte años (1925-1945) a que aludimos fueron años equivocados en muchas cosas e impregnados de un amargo sabor que repercutió en todos los continentes. ¡Ojalá que esta Colaboración a la Bibliografía ayude a acercarnos más a la figura y a la inconmensurable riqueza de la vida y obra de San Agustín y nos cambie el sabor de los dolorosos acontecimientos de aquellos años!

\section{INSTRUMENTOS BIBLIOGRÁFICOS}

1.- $\quad$ HAIN, Ludovicus, Repertorium bibliographicum, in quo libri omnes $a b$ arte typographica inventa usque ad annum MD. Typis expressi ordine alphabetico vel simpliciter enumerantur vel accuratius recensentur. Vol. 1, Pars I, Josepf Altmann, Berlin 1925.

San Agustín ocupa las páginas 244-264 con citación de las diversas obras impresas hasta 1500 , un total de 168 , comprendidas entre los números 1946-2114. Son, pues, los incunables con el principio y el fin de los títulos y demás datos necesarios.

2.- COPINGER, W. A., Supplement to Hain's Repertorium Bibliographicum or Collections towards a new Edition af that Work In two Parts. The frst containing nearly 7000 corrections of and additions to the Collations of works described or mentionned by Hain. Part II, Josef Altman, Berlin, 1926, 510 pp.

Las correcciones y adiciones a los respectivos números de Hain ocupan aquí pp. (55-61) y (83-87). Con ello queda desde el $n^{\circ} 732$ a 
1773 y la cita es mucho más completa, ya que añade los datos que faltaban en el anterior o corrige los deficientemente dados.

3.- $\quad$ Bibliotheca philologica classica. Beiblatt zum Jahresbericht über die Forschritte der klasischen Altertumswissenschaft, begründet von Conrad BURSIAN herausgegeben von Andreas THIERFELD, 52 (1925), nos. 1148-1176; 53 (1926), nos. 1157-1199; 54 (1927) nos. 993 1021; 55 (1928), nos. 1002-1026; 56 (1929), nos. 1434-1491; 57 (1930), nos. 1455-1750; 58 (1931), nos. 1500-1762; 59 (1932), nos. 1430-1515; 60 (1933) nos. 1623-1676; 61(1934) nos. 1645-1697; 62 (1935) nos. 600-653; 63 (1936) nos. 681-722; 64 (1937) nos. 638-673; 65 (1938) nos. 653-704.

Esta ingente colección iniciada en 1884 es un arsenal para la bibliografía agustiniana. Hemos citado nada más los números que dedica a San Agustín a partir de 1925 . Un total de 1140 títulos hasta el 1938. Se han sucedido en su elaboración autores diversos desde Friedrich VOGEL a R. KAISER, Wilhelm RECHNITZ, O. R. REISLAND, Walter ABEL y Gerhard REINCKE. En la colección se dan solamente los títulos de las obras o de los artículos y de ella nos hemos servido en parte para la colección que presentamos, con lectura y resumen que ofreceremos en cada caso.

4.- $\quad$ Gesamtkatalog der Wiegendrucke. Herausgegeben von der Kommission für den Gesamtkatalog der Wiegendrucke. Band III. Ascher Bernardus Claravallensis. Leipzig, Verlag von Karl W. Hiersemann, 1928.

En la rúbrica dedicada a San Agustín, hay una: Agustín, que ocupa las columnas 65-128 y otra el Pseudo Agustín, cols. 128-180. Y en las descripciones del documento se da ordinariamente la información bibliográfica, la colación, la noticia del texto con indicaciones de las fuentes y el elenco de los ejemplares.

5.- $\quad$ NEBREDA, Eulogius, Bibliographia augustiniana, seu Operum Collectio, quae divi Augustini vitam et doctrinam quadantenus exponunt. Romae, Typ. Pol. "Cuore di Maria", 1928.

Se recogen 934 títulos de obras o artículos sobre San Agustín desde el año 423 hasta el año 1928. Incompleta, fue durante mucho tiempo el lugar bibliográfico común de los agustinólogos. Por el número de títulos se adivina que no puede ser exhaustiva, y esto aparece to- 
davía más claro para el siglo XX. De los artículos en general se da solamente el título, a veces también el número y el año de la Revista, pero casi nunca las páginas. Fue un gran paso en la historiografía bibliográfica agustiniana, pero tanto en el método como en el contenida deja mucho que desear para hoy. Su consulta, sin embargo, es siempre provechosa.

6.- $\quad$ BOYER, Charles, Bulletin augustinien, en "Gregorianum" 9(1928), 317-323; 10 (1929), 102-111; 11 (1930), 600-617; 12 (1931), 618-639; 14 (1933), 87-96.

En todos los Boletines, Boyer da una amplia recensión de las obras o de los artículos que analiza, y en ocasiones, emite también sus reservas. En el Primero se entretiene con Cayré, Combés y Sestili. En el Segundo se detiene en Vega, Nebreda, Riviére, McKeough, Sestili y Jansen. En el Tercero se preocupa de la Miscellanea Agostiniana y de las Acta Hebdomadae Augustinianae-Thomisticae, de Gilson, Alves Pereira, Schneider. Y señala las números especiales que han dedicado o piensan dedicar -así lo anuncia- diversas Revistas con motivo del Centenario de San Agustin(1930), Concetti, Fabio di Maria y algún estudio sobre las Confessiones y el De civitate Dei. En el Cuarto da este índice: Miscellanea Agostiniana, Rivista di Filosofia Neoscholastica, Vita e Pensiero, Religión y Cultura, Concetti, Despiney, Comeau, Therése Sullivan, K. Adam,Vega, Hessen, Krebs, Pincherle. Y en el Quinto estudia a Marcos del Río, Vega, Bruceuleri, Jolivet, Montanari, Staszko y Capelle. Obras todas ellas y artículos que merecerán un apunte en su lugar correspondiente.

7.- $\quad$ VEGA, Ángel Custodio, Bibliografía intorno al pensiero filosofico di S. Agostino, en "Bolletino Storico Agostiniano" IV-6 (1928), 179. 184; $V-1$ (1928), 24-26.

Brinda la bibliografía que había recogido en su Introducción a la filosofía de San Agustín.

8.- $\quad$ B., Le principali edizioni di tutte le opere di S. Agostino, en "Bolletino Storico Agostiniano" V-21 (1929)55.

Cita de las ediciones de Basilea, de Erasmo, de Lyon, de Lovaina, Maurina, repetida ésta en Paris, Amsterdam, Venecia, Paris-Gaume, Caillau y Migne y la edición del CSEL. 
9.- $\quad$ BARDY, G., Chronique d'histoire des origines chrétiennes, en "Revue apologétique ", 48/1 (1929), 328-346; 51/2 (1930), 213-234.

Se refiere únicamente a las obras de Slomkowski y lo hace en las $p p$. 337-339 y en el segundo habla de Gilson, pp. 224-226.

10.- DE GHELLINCK, Joseph, Les etudes patristiques depuis 1869, en "Nouvelle Revue Théologique" 56 (1929), 840-862.

Se habla en dos ocasiones de San Agustín con poco interés para su estudio.

11.- DÜRRIES, Hermann, Fünfzehn. Iahre Augustin Forschung, en "Theologische Rundschau", Neue Folge 1(1929), 217-246.

En dos páginas plenas de contenido y de letra diminuta anota una serie de títulos de obras agustinianas, de 1914 a 1929, más o menos. Luego ofrece una visión general de los problemas abordados y de las soluciones dadas a los mismos, acercando las obras de idéntico tema. La enumeración es conducida por orden alfabético de autores.

12.- $\quad$ DUDON, Paul, Bulletin d'histoire ecclésiastique. Politique de saint Augustin, en "Etudes" 200 (1929), 216-219.

Amplia recensión y acogida de la obra de G. Combés sobre la política en San Agustín.

13.- VARIOS, Augustinus, en "Scholastik" 5 (1930), 599-605.

Con recensión y crítica diversos autores recorren las páginas de Lesaar, de Mausbach, de Billicsich, de Jonas, de Backs, de Cavallera, de Boigelot, así como los artículos de los números de 1930 de la "Nouvelle Revue Théologique", de "Gregorianum", de "Studia catholica"; de "Studien", de "Vie spirituelle". En total ocupa del número 331 al 343.

14.- "Bolletino bibliografico ". Storia della filosofta antica. 1930.

15.- $\quad$ SILVA- TAROUCA, P., Intorno alla vita e agli scritti di S. Agostino, en "La Civiltá Cattolica" 81-3 (1930), 152-158.

Un examen no profundo, sino más bien descriptivo de algunas obras sobre san Agustín. Entre ellas el número especial de "Gregorianum", D. Bassi, Concetti, Sgariglia y Papini. 
16.- Catalogo della Biblioteca Agostiniana con illustrazioni. Firenze, Lib. ed. Fiorentina (XV Centenario Agostiniano), 1930, 64 pp.

No de gran interés, pero al menos se pone al corriente de las obras publicadas en italiano en esa "Biblioteca Agostiniana", surgida en 1926 por iniciativa y bajo la dirección del P. Bellandi. Se han editado traducciones de escritos de S. Agustín y algunas obras originales.

17.- BLANCO SOTO, M., Bibliografía agustiniana, en "Archivo Histórico Agustiniano" 35 (1930-1931), 161-164, 307-318, 456-472; 36 (1931), 112-148, 310-320, 464-478; 37 (1932), 307-320, 459-474; 38 (1932), 142-157, 301-316, 465-478; 39 (1933), 303-320, 456-473; 40 (1933), 301-316; 41 (1934), 301-317, 440-471; 42 (1934), 115-146, 274$293 ; 43$ (1935), 107-139, 278-293, 430-462, 607-622.

Modestamente expone sus deficiencias y sus dificultades a un plan majestuoso y soberbio que proyectaba: "intento escribir-dice- una Bibliografia Agustiniana que contuviese: primero, la noticia de todos los manuscritos del Santo que se conservan actualmente en las bibliotecas; segundo, enumerar las ediciones de todas sus obras, tratados particulares y versiones a las lenguas modernas; tercero, formar una bibliografía de todos los escritores que han tratado de la vida, virtudes y escritos del Santo" (p. 162). El catálogo -lo admite- tiene que ser deficiente y defectuoso (p. 163), pero ve que lo es sobre todo en la parte referente a los manuscritos (p. 164), que la publica para que alguien la complete. Y comienza por "Scripta Sancti Augustini", sigue con Obras de San Agustín mencionadas en las Retractationes y que ya no existen". "Escritos de San Agustín mencionados por San Posidio", su índice y por el Venerable Beda y que tampoco existen", "Obras", "Manuscritos", a estos dedica su mayor parte, "Impresos", "Opera omnia", "Opuscula plurima". Y en cada uno va citando con breves anotaciones en español.

18.- DE SANCTIS, Renzo, Bibliografia, en "Studium" 26 (1930), 727 731.

Confiesa que son "notacelle" para quien sea extraño plenamente al tema agustiniano. Como autores principales cita a Nebreda, a Gilson, a Meda, a Pincherle, a Papini, referidas a la vida de San Agusdtín y luego las traducciones de dos obras principales Confessiones y De civitate Dei. Y siguen algunos otros autores como Portalié, Guzzola, Alfaric, Martin, Storz, Mausbach, Roland-Gosselin, Combés, Batiffol, Cayré, Adam. 
19.- JUGIE, M., Saint Augustin dans la litérature théologique de L'Eglise russe, en "Echos d'Orient" 33 (1930), 385-395.

Recopilación de un buen número de obras o artículos sobre San Agustín en la Iglesia rusa bajo estos dos epígrafes: I. Traducción de obras de S. Agustín en lengua rusa; y II. Obras y disertaciones sobre la vida y la doctrina de S. Agustín. 8 forman el número primero y 22 el II'.

20.- $\quad$ KRÜGER, Gustav, Neule Augustinliteratur, en "Zeitschrift für Kirchengeschichte" 49 (1930), 494-501.

Completa algunos boletines de años anteriores y da abundantes, sino exhaustivas noticias de las obras sobre san Agustín de 1929 y 1930. En el texto expone el contenido o una breve crítica de la obra y en nota cita la obra o el artículo.

21.- CAPELLE, Bernard, Chronique de Théologie ancienne. Le XV Centenaire de saint Augustin, en "Recherches de Théologie ancienne et mediéval " 3 (1931), 307-314.

Extrae las conclusiones que brindó el Centenario, una vez que el Boletín recoge las publicaciones y no hace aquí más que remitir a aquellos números. "Ha llegado la hora de rendir cuentas. ¿Qué provecho se ha sacado de este esfuerzo? Ha sido, creo yo, múltiple y no despreciable. Trataremos de extraerlo brevemente en esta Crónica"(p. 307). "La modernidad de Agustín -escribe- es de orden religioso más bien que teológico. El hombre de hoy, cuando lee las Confesiones, se ve tentado a saltar los pasajes doctrinales que interrumpen las confidencias del convertido. Y es que Agustín es moderno menos por la doctrina que porque el alma, en él, supo elevarse por encima de las contingencias inmediatas, para entregarse ampliamente a las emociones humanas, que son de todos los tiempos"(p. 307).

22.- CUERVO, M., Boletín de literatura agustiniana, en "La Ciencia Tomista" 23 (1931), 208-243.

"Nosotros no pretendemos hacer aquí un análisis de todas las obras que con ocasión de este Centenario se han escrito; en primer lugar porque algunas todavía no se han publicado por encontrarse aún abierto un importante concurso, y no pertenecer otras a esta sección por tratarse en ellas de estudios biográficos o de otros aspectos 
menos importantes del gran obispo de Hipona"(p. 208). Divide su amplia recensión en I. Trabajos en forma de libros, y aquí habla principalmente de Gilson, de Mausbach, de Alves Pereira y de Saint -Martín. II. Otros trabajos, en artículos o revistas, haciendo recuento $\mathrm{y}$ análisis de los artículos publicados en revistas, dividiendo los temas en filosofía y teología, teoría de la iluminación, pluralidad de formas y transformismo, la paz y la guerra según san Agustín, existencia de Dios, el método apologético según san Agustín, la gracia, la Trinidad, la ciencia de Cristo, la teología sacramentaria, la espiritualidad de san Agustín, las Retractationes, el porvenir de la metafísica agustiniana.

23.- FERRO, Andrea, Rassegna agostiniana, en "Bilychnis" 36 (1931), 89-102.

Recensión y crítica de unas cuantas obras biográficas y doctrinales. A. Pincherle, Billicsich, Romeis, Krebs, Lesaar, Schäffer, Heusse, Von Bernhardt, Bassi. Después se detiene en algunos artículos de Estudis franciscans, Saint Augustin=Cahiers de la Nouvelle Journée y Vita e Pensiero.

24.- GONZÁLEZ, Raimundo, Bibliografía agustiniana del Centenario, en "Religión y Cultura" 15 (1931), 461-509.

Ha tratado de realizar una labor completa sobre el XV Centenario de la muerte del santo Doctor y recoge 574 títulos, distribuidos en los siguientes epígrafes: I. Ediciones y traducciones, nos. 1-43; II. Vida de San Agustín y obras de carácter predominantemente biográfico, nos. 44-87; III. Estudios: Libros y ensayos, nos. 88-144; IV. Misceláneas, nos. 145-307, y apela a diez misceláneas con los títulos de los artículos que contienen; V. Revistas, números extraordinarios, nos. 308-411; VI. Revistas, artículos sueltos, nos. 412-551; VII. Libros -Homenaje a sabios célebres, nos. 552-558, VIII. Pastorales-. Conferencias y Panegíricos, nos. 559-574. A todo esto agrega un buen número de revistas populares o piadosas, juntamente con algunas citaciones que ha omitido por no haber llegado a tiempo a sus manos, tales como el II "Miscellanea Agostiniana", y la obra del Prof. Montanari "I Massimi Problemi in S. Agostino".

25.- NAIRN, John A., A hand-list of books relating to the classic and classical Antiquity. Oxford Blackwell 1931, 161 pp. 
Breves referencias a San Agustín.

26.- $\quad$ ROMEYER, B., Chronique bibliographique sur la philosophie augustinienne, en "Revue de Philosophie" (1931), 584-609.

27.- La "Bibliografia agostiniana del Centenario" e $i$ "Sermoni di S. Agostino", en "La Civiltá Cattolica" 83-4 (1932), 152-159.

Da noticia de la "Bibliografia agustiniana del centenario", alabándola, y se entretiene luego en el análisis de los Sermones post Maurinos reperti de Morin.

28.- $\quad$ Rassegna di nuove traduzioni delle opere di S. Agostino, en "La Civiltá Católica" 83-3 (1932), 136-150.

Un juicio largo con las reservas que consideran oportunas sobre las traducciones de las Confesiones de A. Masini, G. M. Llovera, de los Soliloquios por Primo Montanari y sobre las páginas escogidas de P. Gerosa, discursos selectos de Ed. Logi y sobre la obra de Bracci.

29.- $\quad$ ARAUJO -COSTA, Luis, Los trabajos agustinianos del P. Vega, en "Religión y Cultura" 20 (1932), 91-95.

30.- $\quad$ KREBS, E., Neuere Augustirrusliteratur, en Theologische Revue 31 (1932), 49-53, 97-106, 137-143.

Divide la recensión en tres partes: 1 . Valoración general y presentación de la evolución religiosa de Agustín, señalando aquí y recensionando 7 títulos de obras. 2. Escritos especiales sobre filosofía y teología de Agustín, señalando 10 trabajos en esta sección. 3. Títulos varios, donde recoge unos 50 bajo epígrafes diversos. En los primeros da crítica y contenido, y en los últimos, ofrece brevemente el contenido con resumen del artículo.

31.- $\quad$ Saggi sulla dottrina di S. Agostino, en "La Civiltá Cattolica" 84-2 (1933), 375-378.

Amplio y elogioso análisis del vol. del P. Boyer "Essais sur la doctrine de saint Augustin".

32.- KRÜGER, Gustav, A decade of research in early christian litterature(1921-1930), en "Harvard Theological Review" 26 (1933), 173-321. Dedica a san Agustín las pp. 292-321 con una bibliografía competente y abundante. Divide las obras del modo siguiente: a) Textos, 
b) Vida, obras y doctrina. En cada apartado da primero la bibliografía en letra pequeña, haciendo luego el comentario o la crítica.

33.- LAFEYRE, G. G., Chronique bibliographique, en "Revue Tunisienne"(1933).

Dice él mismo: "Dans une chronique bibliographique de la "Revue Tunisienne" nous avons donné, en 1933, une liste á peu prés compléte des editions critiquers, traductions, études biographiques, litteraires, philologiques, philosophiques et théologiques qu'ont paru á l'occasion du XV Centenaire de la mort de saint Augustin(430-1930) " (De nouveau sur s. Augustin. p. 196).

34.- STEENBERGHEN, Fernand van, La philosophie de St. Augustin d'aprés les travaux du Centénaire, en "Revue néo-scolastique de Philosophi" 34 (1932) 366-387; 35 (1933), 106-126, 230-281.

"Ha llegado la hora de cosechar y entrojar" (p. 368). Y luego de pasar revista a las diversas bibliografias del XV Centenario con algunos reparos a las mismas, expresa así el plan de su trabajo: "Queríamos, limitándonos al dominio de la filosofía, coordinar y completar los resultados adquiridos. No hace falta decir que no se tendrán en cuenta la literatura de circunstancias y de vulgarización; y en cuanto a los trabajos científicos, nos detendremos en la medida en que presenten un interés filosófico. Para realizar lo mejor posible este diseño me ha parecido de gran provecho distinguir en la exposición que va a seguir, una sección propiamente bibliográfica y una sección doctrinal. La primera tiene por objeto la descripción y el análisis sumario de la literatura agustiniana reciente; luego de haber hecho conocer las publicaciones colectivas del Centenario, presentamos las principales obras aisladas que han sido consagradas a san Agustín en el curso de los últimos años. Hecho esto, podremos con mayor libertad en la sección doctrinal, entregarnos al examen de los temas capitales de la filosofía agustiniana a la luz de los trabajos recientes y desprender de nuestra encuesta algunas reflexiones sobre el agustinismo" (p. 369). Desarrolla el plan como lo ha planteado: Publicaciones Colectivas, Obras aisladas con estos apartados: 1. Trabajos históricos y biográficos; 2 . Ediciones e investigaciones de crítica literaria; 3. Obras doctrinales. En los artículos siguientes: temas capitales de la filosofía agustiniana, la filosofía, los problemas y los métodos, la crítica del conocimiento, la metafísica. del conocimien- 
to, el alma y el cuerpo, los grados del conocimiento humano, con una conclusión general sobre el agustinismo (pp. 272-281).

35.- STOHR, Albert, Aus der neuesten Augustinus forschung, en "Theologische Revue" 33 (1934), 481-486.

Recensiona tres obras: Verwiebe, Theiler y Dinkler. Copia el índice de la obra, hace una exposición y orienta diciendo para qué sirve en concreto.

36.- Gesamtkatalog der Preussischen Bibliotheken. "Augustinus". Herausgegeben von der Preussischen Staats bibliothek Berlin, Preussische Druckerei und Verlagaktiengesellschaft, 193 5, y "Speculum" (1935), 477-588.

37.- MANITIUS, Max, Handschriften antiker Autoren in mittelalterlichen Bibliothekskatalogen von-. Herausgegeben von Karl Manitius. 67. Beiheft zum Zentralblatt für Bibliothekswesen. Leipzig, Otto Harrassowitz, 1935, 357 pp.

A san Agustín dedica, las pp. 224-225, recogiendo los manuscritos de los catálogos de las Bibliotecas medievales, sean alemanas, francesas, inglesas, italianas o españolas.

38.- THEILER, Willy, Augustin, en "Gnomon" 11 (1935), 36-41.

Recensión, comentario y anotaciones a las obras de C. Y. Balunus, M. Cousin, K. Kaypers, K. Svoboda, R. Jolivet.

39.- VEGA, Ángel Custodio, Boletín agustiniano, en "Religión y Cultu$r a " 29$ (1935), 223-234. En su forma habitual.

40.- $\quad$ WULF, Maurice de, History of Mediaeval Philosophy. Translated by Ernest C. Messenger. Vol. I. From the Beginning to Albert the Great. London - New York, Longmans, Green and Co. Ltd., 1926, XVI-416 p., $3^{\text {a }}$ Ed. 1935.

Estudia la filosofía de san Agustín en las pp. 115-121, y en diversas partes remite a su doctrina y la expone, de acuerdo a la historia.

41.- ' MERLAN, Philipp,1.W.Theiler, Porphyrios und Augustinus - 2. J. Guitton, Le temps et Z'eternité chez Plotin et saint Augustin - 3. P. Henry 
Plotin et l'Occident - 4. J. Barion, Plotin und Augustin, en "Gnomon" 12 (1936), 527-543.

El neoplatonismo de Agustín y sobre todo el influjo de Plotino o de Porfirio desvelado a través de estas obras. Resume los estudios y ofrece sus conclusiones.

42.- $\quad$ ROMEYER, Blaise, Bulletin de philosophie chrétienne. I. Période patristique, en "Revue de Philosophie" 12 (1936), 501-549.

Dedica unas páginas a san Agustín.

43.- Bibliographie de philosophie. Institut Internationale de collaboration philosophique. Paris, J. Vrin, 1937,I-1 (1937), 187 pp.; I-2 (1937). Paris, J. Vrin, 1938, 343 pp.; II-1(1938), Paris, J. Vrin, 1938, XII-247 pp.; II-2(1938), Paris, J. Vrin 1939, IX-341 pp.; III-1 (1939) Paris, J. Vrin, 1944, VI-330 pp.

En todos ellos a la palabra "Augustinus (Aurelius)" pueden verse señalados, nada más algunos estudios, obras o artículos sobre el santo. Incompleto, pero útil.

44.- $\quad$ BARDY, Gustave, Chronique d'histoire des origines chrétiennes. 3. Saint Augustin, en "Nouvelle revue Apologétique" 2 (939), 167-172.

45.- WOLF, Ernst, Zur neueren Augustinusliteratur, en "Theologische Blätter" 18 (1939), 177-190.

Notas a unas cuantas obras sobre diferentes temas agustinianos. Expone el contenido de la obra y critica lo que cree conveniente. Los autores son los siguientes: F. Hofmann, H. Eger, Lewalter, Dinkler, Platz, K. Janssen, H. Barth, Hendrickx y otros.

46.- CHERUBELLI, Paolo, Le edizioni volgari delle opere di S. Agostino nella rinascita. A cura e con introduzione di (Biblioteca agostiniana, 42). Firenze, Tip. Fiorentina, 1940, 150 pp.

Interesan las dos primeras partes, que recogen respectivamente los escritos en italiano referentes a las obras de San Agustín y a la Regla. Se trata no sólo de impresos, sino también de manuscritos. Da la cita completa y una breve reseña del escrito.

47.- $\quad$ KAMPMANN, Theoderich, Augustinus deutsch, en "Theologie und Glaube" 34 (1942), 268-270. 
Recuento de algunas traducciones, de Heffele, Confessionum libri, de Perl, De musica, De ordine, De libero arbitrio.

48.- HERESCU, N. I, Bibliographie de la littérature latine. Paris, Les Belles Lettres, 1943, XVIII-426 pp.

Bajo el título Aurelius Augustinus se ofrecen una gran cantidad de obras y artículos que ocupan las pp. 395-410. Se aúnan en torno a manuscritos, ediciones generales, ediciones parciales, extractos, traducciones, estudios, recurriendo en este último apartado tanto a lo doctrinal como a lo histórico referente a la lengua latina y a la filología. La colección se detiene, nos dice el autor, a principios del año 1940.

49.- STEUERT, Hilary, Two Augustinian Studies, en "The Dublin Review" (London), 216 (1945), 61-73.

50.- Bibliographie linguistique des années 1939-1947. (Linguistic Bibliography for the years 1939-1947) Publié par le Comité International permanent de linguistes avec une subvention de l'U.N.E.S.C.O, t. I: Utrecht - Bruxelles, Spectrum, 1949, 237 pp.

51.- VON CAMPENHAUSEN, H., Neuere Augustin - Literatur I., en "Theologische Runschau" N. F. 17 (1948-1949), 51-72.

Abrigaba la intención de completar a Dörries, partiendo desde el año 1929. Excluye biografías y recensiones. Agrupa las obras bajo diversos temas, expone el contenido, descubre las polémicas y orienta bien al lector.

52.- DE BRIE, G. A., Bibliographia philosophica 1934-1945. Vol. I. Bibliographia Historiae Philosophiae, 1950. Vol. II. Bibliographia philosophiae, 1954. Bruxelles, ed. Spectrum, 1950-1954, 2 vols., LXXV664, XXXII-798 pp.

"Esta bibliografía se limita a reunir las reseñas de obras filosóficas en alemán, castellano, catalán, lenguas escandinavas, francés, holandés, inglés, italiano, latín y portugués durante el espacio de tiempo comprendido entre los años 1934 y 1945, ambos incluidos " (p. XXI). A San Agustín dedica en el vol. I, las pp. 115-126, ocupando los números 4008-4364, reunidos bajo los epígrafes generales: I. Opera et scripta de operibus Augustini, II. Scripta historica et historico-doctrinalia; III. Monographiae secundum diversitatem doctrinarum. Y 
en este último apartado distingue: metafísica, cosmología-biología, psicología-crítica, ética, lingüística, pedagogía, estética. Abundante, rica, muy completa, aunque sin contenidos ni recensión, sólo cita las ouras o artículos.

53.- BIGNAMI ODJER(J.) -BROU (L.)-VERNET(A.), Bibliographie sommaire des travaux du Pére André WILMART O.S.B. 1876 -1941. (Sussidi eruditi,5). Roma, Edizioni di Storia e Letteratura 1953, 144 pp. y Bibliografia, pp. 31-79.

Se recogen al menos 24 trabajos que interesan al estudioso de san Agustín, de modo especial los manuscritos de sus obras y más particularmente los Sermones. De ellos se dará nota en lugar oportuno. Ese número de estudios va del año 1925 al 1941 año de su muerte.

54.- DEL ESTAL, Juan Manuel, Historiografía de la "Ciudad de Dios", 1928-1954, en "La Ciudad de Dios" número extraordinario Estudios sobre la "Ciudad de Dios" II, 1955, El Escorial, 647-774.

Da un total de 404 títulos concernientes a la Ciudad de Dios y su problemática. Indispensable para quien pretenda estudiar un tema de esa obra. Los ha reunido en torno a estos temas. I. Ediciones críticas. II. Traducciones. III. Antologías. IV. Estudios. Breve resumen del artículo o recensión tornada de otra revista.

55.- $\quad$ COUSIN, Jean, Bibliographie de la langue latine 1880-1948. (Collection de Bibliographie Classique). Paris, Les Belles Lettres, 1951, 375 pp.

La obra no tiene gran interés para el estudio de san Agustín. Se citan algunos artículos u obras sobre la lengua y la filología agustinianas en las páginas siguientes $128,150,156,186,173,203,207,214$, 215, 217, 230, 236 sobre todo aquí bajo el nombre de Augustinus, 280 anteriores al 1923, 303, 311 y 333 anteriores al 1925 .

56.- VALVEKENS, J. B., Augustirrusstudién tijdens de laatste 25 jaar, en "S. Augustinus ". Bij het XV eeuwfeest van zijn geboorte 354-1954. Averbode, ed. "Pro nostris", 1954, pp. 9-24.

Promete más el título que da la realidad. Un análisis somero de las principales obras, de las corrientes de interpretación, de las nuevas revistas agustinianas. Es más bien un ensayo sobre el movimiento 
agustiniano que un estudio o recuento bibliográfico de los 25 últimos años.

57.- $\quad$ LAMIRANDE, Emilien, Un siécle et démi d'études sur l'eclésiologie de saint Augustin. Essai bibliographique, en "Revue des Etudes Augustiniennes" VIII-1 (1962), 1-125.

$\mathrm{El}$ autor recoge para los años que nos interesan los nos. 353-762, de 1925 a 1949 ambos inclusive. Señala ordinariamente en la breve recensión que hace las páginas que los autores dedican a San Agustín, de modo especial a la eclesiología y temas afines. La selección no es completa y hay mucha abundancia en el tema, y es muy completa la cita.

\section{MISCELÁNEAS}

58.- $\quad$ La Settimana Agostiniano-Tomistica, en "La Civiltá Cattolica" 81-2 (1930), 360-367.

Una acrobacia periodística y expositiva del desarrollo de la Semana y su contenido doctrinal.

59.- $\quad$ Saint Augustin. Cahiers de la Nouvelle Journée, 17. Paris, Librairie Bloud et Gay, 1930, 208 pp. (Saint Augustin, París).

Artículos de M. Blondel, M. Comeau, J. Romand, J. Chaix-Ruy, E. Castelli, J. de Pange, Ch. Boyer, y un suplemento sobre las ideas y los libros debida a la pluma de P. Archambault, J. Nanteuil, P. Le Cormier, B. Amoudru, que ocupa las páginas 103-205.

60.- Saint Augustin. Sa sainteté, sa doctrine spirituelle. (Les grandes mystiques). Extrait de "La Vie spirituelle" 1930. Juvisy (J.- et - O.), Editions du Cerf, 1930, 157 pp. (La V.S.1930).

Tras un resumen de "las principales verdades que pueden esclarecer mejor nuestra vida interior", extraídas de la Enc. Ad salutem humani generis en un artículo sin firma titulado "A la gloire de saint Augustin"(pp. 5-14), hay colaboraciones de A. M. Jacquin, P. de Labriolle, Possidius, B. Roland - Gosselin, R. Garrigou-Lagrange, J. Riviére y M. D. Chenu.

61.- St. Augustin, 430-1930. Zur Jahrhundertfeier dargeboten von der Deutschen Provinz der Augustiner Eremiten. Würzburg, St. RitaVerlag, 1930, 265 pp. (= St. Augustin Würzburg). 
Dividido en tres partes: Vida, obras e hijos espirituales, con artículos breves de Beckmann, de E. Eberhard, B. Leonhardt, R. Arbesmann, H. Appelhaus, N. Greubel, Max Rüssler, H. Seller, P. Vollmer, D. Spitzenberg y precisiones interesantes.

62.- Augustiniana. Dissertationes et Orationes habitae in celebrationeAnni Jubilaei S. Augustini diebus 7ma. et 8va. Augusti 1930 in Abbatia Averboiensi Ordinis Praemonstratensis. Averbode, edita a Commissione "Pro Hostia", 1930, 168 pp. (=Augustiniana - Averbode).

Contiene dos partes: I. Dissertationes habitae in sessionibus academicis: de Joanes Baptista Valvekens, Gelacus Hoste, Paulo Lenaerb, E. Gisquiere, Hugo Hejman; II. Orationes habitae in ultima solemnioreque sessione: de Gummarus Crets, Hugo Lamy, Hippolytus Gergye, E. Gesquiere, con un proemio dedicado a ensalzar la fecha que se conmemora.

63.- GISQUIERE, Emmanuel, Sanctus Augustinus et laus Dei, en "Augustiniana, Averbode" 1930, 164-8.

Le queda alabar a Dios y lo hará quo Augustino et cum Augustino.

64.- CRETS, Gummarus, Auspicalis allocutio, en "Augustiniana, Averbo$d e " 1930,129-130$.

Ensalza el ejemplo de Agustín para la Iglesia y para la Orden de los Premonstratenses.

65.- LAMY. Hugo, Oratio panegyrica S. P. Augustini, en "Augustiniana, Averbode" 1930, 131-156.

Presenta el alma de Agustín, se deteniene ante su imagen y hace algunas reflexiones. "Nobis enim repraesentatur S. Augustinus una manu librum tenens, ac cor igne amoris flagrans, ex altera vero, baculum ferens pastorale" (p. 135). Ciencia del Doctor y solicitud pastoral.

66.- GERGYE, Hyppolitus, Oratio panegyrica S. P. Augustini, en "Augustiniana, Averbode" 1930, 157-163.

Extrae las lecciones del cuadro de Schäeffer: "Sanctus Augustinus, considens, pectori materno placide adhaerescit, vultum a regionibus patriae cujus desiderio ictus, longo itinere faciendo accingitur, non removet, at -videte- oculos in alta tollit" (p. 162). 
67.- Augustiniana. Nel XV Centenario dalla morte di S. Agostino. 4301930. Napoli, tip. R. Contessa \& Fratelli, 1930, 142 pp. PP. Agostiniani prov. di Napoli (= Augustin. Napoli).

Una serie de cartas congratulatorias del Rvdmo. P. Eustasio Esteban y de diversos Cardenales inicia la publicación con un Carmen saeculare dedicado a S. Agustín por Iosue CACALE(pp. 13-15). Y siguen artículos de E. Cocchia, F. Meda, A. Addeo, N. Barone, M. de Martino, D. Bassi, G. Lepore, C. Cirillo, P. Ciuti, M. Autore, S. Bellandi, L. Teixidor, A. Tonna-Barthet, G. Faraoni, G. Abruzzese, E. A. Fabozzi, G. della Rocca y S. Procaceino. Reproduce diversos cuadros y una página de "Il Figlio delle lagrime". Oratorio per soli, coro e orchestra del P. C. Crispo (p.143).

68.- Aurelius Augustinus. Die Festschrift der Görres-Gesellschaft zum 1500 Todestage des hl. Augustinus, heraugegeben von Martin GRABMANN und Joseph MAUSBACH. Köln, Verlag J. P. Bachem, 1930, XI-438 p. (=Aurelius Augustinus).

Tras unas palabras de presentación(pp. VII-VIII) de J. Mausbach, siguen dos páginas (p. IX-X) de J. Sauer sobre la "imagen o pintura del título" (zum Titelbild). Luego hay artículos de Allgeierl, Dyroff, Geyser, Grabmann, Jansen, Jedin, Kunzelmann, Mausbach, Merkle, von Rintelen, Romeis, Sauer, Schilling, Schmaus. Schmitt, Sühngen, Stogmüller, Vogel.

69.- Cultura Social 212(1930), Manila.

Número dedicado casi en su totalidad a S. Agustín, ocho artículos con varias ilustraciones.

70.- $\quad$ Souvenir of the Fifteenth Centenary of the death of St. Augustin 4301930. Augustinians of the American Province s.f., 78 pp. (=Fifteenth Centenary).

Contiene breves estudios de E. Tourscher, la Enc. "Ad salutem humani generis" de Pío XI (pp. 19-51) en traducción inglesa, de E. A. Foran, con otro apunte sobre los eremitas de san Agustín y la labor de los agustinos en América del P. Francis Xavier Roter.

71.- Alfonso XII. Publicación de los Alumnos del Real Colegio de Alfonso XII de El Escorial. Año VI, núm. 6, junio de 1930. Al gran 
Padre y Doctor de la Iglesia San Agustín en el XV Centenario de su muerte, 226-356 pp.

En las pp. 231-268 dedicadas a san Agustín hallamos de la redacción Eı el XV Centenario de la muerte de S. Agustín, y artículos debidos a los autores jóvenes, en su mayor parte estudiantes de bachillerato: C. V., San Agustín estudiante (pp. 233-237); Félix RODRÍGUEZ, La conversión de san Agustín (pp.238-241); C. RUSIÑOL VACA, Pensamientos de san Agustín (pp. 242-246); Mariano de LAS PEÑAS, Elogios a san Agustín (pp. 247-250); R. S. M., Los últimos dias de San Agustín (pp. 251-254); $V$. RUSIÑOL VACA, Obras de San Agustín (pp. 2S1-2S4); C. V. M., Las reliquias de San Agustín (pp. 258-262), V. CAÑAS CIRULO, Iconografía de San Agustín (pp. 263264). Además se ofrece alguna poesía sobre el Santo.

72.- Ambrosius. Bolletino liturgico ambrosiano. VII, n. 11. Numero speciale dedicato a S. Agostino, pp. 201-264. Milano 1930. (=Ambrosius).

Artículos de Dotta, de Bernareggi y de Vatova.

73.- $\quad$ Archives de Philosophie, Volume VII - Cahier II. Etudes sur sait Augustint (430-1930). Paris, Beauchesne, 1930, 272 pp. (=Archives de Philosophie).

Con artículos de R. Jolivet, Ch. Boyer, F. Cavallera, B. Romeyer, R. de Sinety, P. Munnot.

74.- $\quad$ Festspáel zum 1500 Todesjahr des Heiligen Augustinus in der Altehrwürdigen Augustiner- kirche zu Beuron - Am Sommerhochfest des heiligen Benediktus 11. Juli 1930, 15 pp.

Una especie de juego (comedia) litúrgico en que hacen intervenir con breves párrafos al recitador $=$ praeco, Posidio, Cristo y san Lucas, y de otra parte, Agustín, seis cantores, el ángel de Agustín y un bromista. Y celebran la conversión y el bautismo, el presbítero y el obispo, la muerte y su gloria celeste. El texto está en latín y alemán, y alternan los cantores e interlocutores, concluyendo con San Benito conmemorando su día.

75.- Documentation Catholique 24(1930). Le Centenaire de saintAugustin. Paris, 1930, cols. 257-302. (= Documentation catholique). Con actas del episcopado, del cardenal Segura, del card. Schuster y Mgr. 
Durand, además de artículos de G. Goyau, de F. Cayré, de F. van den Kornhuyse, de P. Tranquile y L. Meyer.

76.- Décimo Quinto Centenario de la muerte del eximio Doctor de la gracia san Agustín, 430-1930. Quito Ecuador, edit. Ecuatoriana, 1930, 238 pp. (Centenario Quito).

Poca valía de lo ofrecido y mucha valentía en el elogio.

77.- Fünfte Lektorenkonferenz der deutschen Franziskaner für Philosophie und Theologie. Schwarz in Tyrol 3 - 7 september 1929. Kloster Sigmaringen - Gorheim (Hohezollern). Franziskus Druckerei WedlWestf. 1930, 218 pp. (Fünfte Lektorenkonferenz).

Tras una introducción y una presentación, contiene artículos relacionados sobre todo con el sobrenatural, de J. Delazer, de C. Romeis de L. Meier, de T. Soirou, y de E. Schlund. Otros artículos se refieren a san Buenaventura y a la actividad de los franciscanos.

78.- Gregorianum, XI-1 De S. Augustino. Romae, 1930, 158 pp. (=Gregorianum).

Colaboración de P. Galtier, Ch. Boyer, B. Leeming A. Vermersch, A. D'Alés, B. Jansen.

79.- Il XV Centenario Agostin. 430-1930. A cura dei RR. PP. Agostiniani Scalzi di Genova. 1930, 47 pp.

Breves artículos sobre la vida, la obra y el pensamiento de san Agustín. Por ejemplo, E. BADINO, Sant' Agostino (pp. 1-4); $R$. NESSI, La gioventú di Sant 'Agostino (pp.79): Q., Il pianto dell' Orto (pp.10-11); Ricordando la morte di Sant'Agostino (pp.12-14); Giulio MARCHI, Sant'Agostino e 1'Impero di Roma (pp.1-20); Giovanni SEMERIA, La Romanitá di sant' Agostino (pp. 21-24); T., La paternitá spirituale (pp.25-26). Más algunos apuntes sobre las familias agustinianas y otros temas.

80.- Augustinus. Feestnummer van de "Studia Catholica". Nieuwe Reeks van "De Catholiek" - Zes de Jaargang - Aflevering IV - Mei 1930, Nijmegen, 231-375. Con artículos de:

C. Smits, H. van Lieshout, Fr. Ferm, J. van den Grintea, J. Schrijnen, Des. Franses, J. de Jong. 
81.- ROSAS Y ESPINAS. XV Centenario de S. Agustín. 430-28.8. 1930. Buenos Aires, 1930, 276 pp.

Dedicado a san Agustín y a los agustinos en Argentina y otras partes del continente americano, con algunos artículos interesantes como: Bonifacio MATA, San Agustín: su juventud y doctor de la Iglesia (pp. 155-159); Indalecio CUENA, Agustín pagano, Agustín santo (pp. 160-164); Aurelio MARTINEZ, Lecciones agustinianas (pp. 16S170); Luis CAMBLOR, Las Confesiones (pp.171-176); Ricardo LLAMAS, San Agustín menos estudiado que nombrado (pp. 178-181); Esteban PORTU, Anhelos del alma de San Agustín (pp. 182-186), Luis GALENDE, El ocaso de una vida (pp. 187-190). Iniciado con una introducción a la Enc. "Ad salutem humani generis" y a un "Auto" sobre el XV Centenario debido al arzobispo de Buenos Aires.

82.- $\quad$ Miscellanea Agostiniana. Testi e studi pubblicati a cura dell ' Ordine Eremitano di S. Agostino nel XV Centenario della morte del Santo Dottore. Vol. L Sancti Augustini Sermones post Maurinos reperti probatae dumtaxat auctoritatis. Romae, Tip Vaticanis, 1930. XII-848 pp. Vol.II.- Studi Agostiniani. Romae, Tip. Vati. 1931, XXXIV-1042 pp. (=Miscellanea Agostiniana).

Recensiones: F. ANTONELLI, Miscellanea, en "Antonianum" 6 (1931), 75-80; A. MANSER, Krönung des Augustinusjahres 1930 durch dom Germain Morin, en "Bibel und Liturgie" 22/23 (193 01931), 1-4 (en extracto); La "Misceldanea Agostiniana ", en "La Civiltá Cattoláca" 83-1 (1932), 448-463; La "Bibliografía agostiniana del Centenario" e I "Sermoni di S. Agostino", en La Civiltá Cattolica" 83-4 (1932), 152159; P. GEROSA, "Sancti Augustini Sermones post Maurinos reperti", en "Convivium", gennaio- febraio (1931), 74-92; C. MOHLBERG en "Ephemerides Liturgica" 46, n. s. 6(1932), 517-519; I. GIORDANI, Sant' Agostino inedito, en "Fides" 30 (1930), 366-369; Ch. BOYER, Bulletin Augustinien, en "Gregorianum" 4 (1930), 600-602; S. COLOMBO, en "Il mondo classico" 1(1931), 9-10; U. B., en "Il pensiero missionario" 3 (1931), 6-8 extracto); A. D'ALÉS, Bulletin de Théologie historique, en "Recherches de science religieus" (1931), 205-207; O. TESCARI, "Rivista di Filologia e di Istruzione Classica, 10(1932), 1-6 (extracto); J. STIGLMAYR, "Zeitschrift für Aszese und Mystik" S (1930), 373-374; G. 
DE LUCA, La Miscel. Agostiniana, en "L'Avenire d'Italia" 17. 9.1930, p. 3, cols. 1-4.

83.- Miscellanea Augustiniana. Gedensboek samengestelduit verhandelingen over S. Augustirrus bij de viering van ziijn zalig overlijden vóór 15 eeuwen CDXXXMCMM. Met 32 Afheeldingen. Uitgegeben door de PP. Augustijnen der Nederlansche Provincie. Rotterdam Brussel, 1930, XXIX - 579 pp. (=Miscellanea Augustiniana.). Unas palabras de Introducción de W. S. Jurgens (pp. IX-X) y otras de agradecimiento debidas a Huib Luns (pp. 578-579) con artículos de S. Th. Makay, Agnes, C. de Brouwer, A. B. Vogel, F. van den Kronhuysen, C. H. Lambermond, P. Hendrikx, D. Franses, H. Robbers, I. Onings, B. H. D. Hermesdorf, K. Smits, W.J. M. Mulder, H. H. Lesaar, J. A. Davids, J. Huyben, B. Jansen, Fr. Sanders, J. G. R. Ter Haar, W. de Vreese, J. Hessen, G. de Reynold, A. J. Claesen, A. Wilmart, Hans Eibl, B. H. J. Weersenbeokin, J. de Vathaire, B. H. Molkenbaer, H. Reul, T. Brandsma, B. Xiberta. Y contiene además 32 ilustraciones.

84.- Gedenkboek bij het vijftiende eeuwfeest van den h. Bisschop Augustinus, Kerkvader, Ordestichter, Steun der Armen -430-28 August 1930. Bewerkt door Belgische Augustijnen, Gent, 1930, 233 pp. (=Gedenkboek, Gent).

Dedicado al Cardenal Van Roey, hace la presentación de las festividades que la Provincia Agustiniana de Bélgica celebra en honor de san Agustín. Tras una breve Introducción (pp. 14-16) del Provincial, P. Prosper Jansen, comienza un estudio biográfico de Agustín a grandes rasgos y a modo de presentación (pp. 16-23). Y se inicia aquí: Fe y amor de san Agustín, pasando luego a hablar de la fe de Agustín (pp. 23-36), de las errores combatidos, maniqueísmo, donatismo, arrianismo, pelagianismo y semipelagianismo (pp. 37-38). En el estudio ya propiamente doctrinal se analiza: I. De la infidelidad a la fe (pp. 42-47); II. Fe en Dios y en su Hijo Jesucristo, fe y razón(pp. 47-52); III. El conocimiento de los misterios de Dios; IV. Los sacramentos, V. La Iglesia de Cristo. Sigue un artículo sobre la vida a través de la Regla, continuándose esta Miscelánea que no se firma con $\mathrm{H}^{\mathrm{a}}$. de la Orden, principalmente en Bélgica.

85.- A Monument to Saint Augustine. Essays on some Aspects of his Thought written in Comme-moration of his 15th Centenary. London, 
Sheed and Ward 1930, 367 pp. Reedited in 1945 (=A Monument to saint Augustine, London).

Artículos de M. C. D'Arcy, M. Blondel, Ch. Dawson, E. Gilson, J. Maritain, C.C. Martindale, E. Przywara, J. B. Reeves, B. RolandGosselin, E. I. Watkin, y una nota previa del compilador, un Epinicium Augustini e índices finales.

86.- $\quad X X X e$ Congrés Eucharistique International. Carthage 1930. Actes et Documents. Tunis, Ed. De la Tunisie catholique, 1931 (Actes et documents).

87.- $\quad$ Akademische Bonifatius. Korrespondenz 45/ 1-2 (1930), 42 pp. y pp. 49-102.

Con artículos de J. Lortz, de T. Steinbüchel, H. Eibl, P. Simon, H. Klein, A. Dempf, B. Poschmann, de R. Grosche, de A. Donders, de valor todos ellos en lo agustiniano.

88.- Philosophia Perennis. Abhandlungen zu ihrer Vergangenheit und Gegenwart. Festgabe Joseph GEYSER zum 60. Geburtstag, hrsg. Von Fritz -.Joachim von RINTELEN. Regens-burg, J. Habbel, 1930, 2 vols. XVIII-525 - X- 525 a 1244 pp. (=Philosophia Perennis).

Para el estudio de San Agustín interesan directamente dos artículos, uno de A. Mayer, de Agustín como místico, y otro de Millar del vol. I, e indirectamente el artículo de De Ghellinck.

89.- Observatorul.- Sfautul Augustin 430-1930. Numar Jubilar al Revistei "Observatorul". Beius Anul III (1930), nos. 4-5-6. Tipauel Tipografici "Ateneul", Beius, 1930, 177-328. Revista rumana dirigida por los PP. Asuncionistas.

90.- Religión y Cultura, 15. XV Centenario de la muerte de San Agustín 430-1930. El Escorial, 521 pp. (Religión y Cultura).

91.- Revue de Philosophie 4-5-6 (1930). XVe Centenaire de la morte de saint Augustin. Paris, 1930, 331-741 pp. (=Mélanges augustiniennes). Artículos de F. Cayré, R. Jolivet, Ch. Boyer, B. Roland-Gosselin, X. Arquilliére, J. de la Riviére, R. Carton, C. G. Thery, E. Gilson, J. Maritain. 
92.- $\quad$ Studiën. Augustinusnummer 113(1930), 273-404. (= Studiën). H. Duurkens introduce y presenta el volumen(pp.273-275), y luego escriben Jan van Ginneken, A. D'Alés, F. Tunmers, E. Hüffer, H. Robbers, A. Slijpen, L. Steger.

93.- Vida nueva, Número extraordinario 28 de agosto 430-1930. Michoacán, Tipografía de Salvador Jurado, 1930,171 pp.

En medio de poesías y dramas escenografiados, se da cuenta de las alocuciones, sermones y conferencias habidas en Méjico con motivo del Centenario XV de la muerte de san Agustín. Un sermón del obispo Luis Martínez y otro de Mons. Luis J. Sepúlveda y una alocución del P. Angel Zamudio y otra del P. Vega, prior de Durango y conferencias del jesuita P. Mariano Cuevas.

94.- Studium, vol. XXVI, n. 10-11-12. S. Agostino nel XV Centenario della morte. Roma, Federazione universitaria cattolica italiana, 1930,581734 (= Studium). Colaboran en este número I. Righetti, G. Mortan, M. Cesaro, V. Padovani, I. Giordani, E. Carboni, F. Montanari, A. Gotelli, M. Perito, E. Mazzantini, S. Van-Rovighi, E. Preto, G. Borgnezio, F. Gatti, A. Grazioli, G. B. Montini R. de Sanctis. Con este programa: I. La vida; II. Las obras; III. El filósofo; IV. El teólogo.

95.- Vita e Pensiero. XV Centenario di S. Agostino 430-1930. Numero commemorativo. Vita e Pensiero. Vol. n. s. XXI, n. 819. Milano, 1930, 450-566 (=Vita e Pensiero). Artículos de A. Gemelli, G. Semeria, F. Olgiati, F. Meda, G. Busnelli, P. Rotta, C. Butli, U. Lattanzi, E. Fabozzi, G. dalla Torre, U. Mariani, A. Cojazzi. Precedidos de unas páginas tituladas: Nella luce di sant'Agostino(pp. 451-457), firmadas por la redacción en que se hace un resumen o indicación de la dirección que llevan los artículos que siguen.

96.- Thomistisch Tijdschrift voor Katholiek Kulturleven. Augustinusnummer 1(1930). Leuven, Dominikanerkloster,1930, 593-778 pp. (Thomistisch Tijdschrift). Artículos de St. Axters, S. M. Zaib, Y. D. Maes, L. Visman, Titus Brautsma, Inn. Wilderbeek, H. van Lieshout, R. J., C. van Gestel, P. J.

97.- $\quad$ Acta Hebdomadae Augustinianae-Thomisticae ab Academia Romana S. Thomae Aquinatis indictae, recurrente XV Centenario anno a 
felici transitu S. Augustini ac simul quinquagessimo anno a creatione ejusdem Academiae. (Romae 23-30 aprilis 1930). Taurini et Romae, Marietti, 1931, 344 pp. (=Acta Hebdomadae AugustinianaeThomisticae).

Se recoge al principio la Enc. Ad salutem humani generis. Luego, muchos artículos estudian temas agustinianos o sus relaciones con los tomistas, así los artículos de Lepicier, Gilson, Casamassa, Boyer, Grabmann, Théry, Laurenti, Garrigou-Lagrange, Sestili, Parisiis, De Simone, Xiberta.

98.- $\quad$ S. Agostino. Pubblicazione commemorativa del XV Centenario della sua morte. Milano, Vita e Pensiero, 1931, 510 pp. e 2 pl. (= S. Agostino). A cura della Facoltá di filosofia dell' Universitá del Sacro Cuore. Escritos de Agostino Gemelli, A. Masnovo, Paolo Rossi, Mario Casotti, Romano Amerio, Silvio Vismara, Francesco Pelluzza, A. Galli, U. A. Padovani, Andrea Oddone, Francesco Olgiati, Melchiorre Roberti, Emilio Abertario, G. Soranzo, Aristide Calderini, Carlo Calcaterra, Giorgio nicomedi, Giovanni Galbiati. Precede la Enc. Pío XI, Ad salutem humani generis.

99.- $\quad$ A Sant' Agostino, Vescovo e Dottor Massimo della Chiesa nella fausta ricorrenza del $X V$ Centenario della sua gloriosa morte $C D X X X$ $M C M X X X$. La Redazione del Periodico "S. Rita". O. D. C. Malta, Valleta, Tipografia Chretien, 1931, 93 pp.

El sumario es amplio y el contenido de los artículos breve. Tras unos pensamientos de diversos obispos, hay artículos de los PP. Giovanni GENOVESE: S. Uistin kabel il conversioni (pp. 11-14); Agostino GEMELLI, La grandezza di S. Agostino (p. 15); Mons. Luigi FARRUGIA: Epigrafe (p. 16); Agostino CARUANA, Santa Monica, Omm S. Uistin (pp. 17-19); Luigi ATTARD, S. Uistin u li spiritu ta penitenza u sacrificciu (pp. 21-24); Francesco GALEA, Santu Uistin $u$ is - salvazioni ta l'eruieh (pp. 25-26); B. PIROTTA, S. Uistin Fundatur ta l'eremiticu Ordni Agostinian (pp. 27-31); Lorenzo M. AGIL7S, L'ulied Spiritualita S. Uistin (pp. 33-35); A. TONNABARTHET, Spirito della Regola di S. Agostino (pp. 37-38); Paul SPITERI, S. Uistin u l'Ghorrief tal Cnisia (pp. 43-44); Evodio BONNICI, Inscriptio latina (p. 45); Mons. Enrico DANDRIA, S. Uistin u li Scrittura mkaddsa (pp. 49-SI); Luigi CATANIA, S. Uistin bhala iskof (pp. 47-48); Mons. Alfonso HILI, S. Uistin u l' Eucaristija (pp. 
55-57); Pius M. AGIUS, Il kalb ta S- Uistin (61-63); Benedetto FSADNI, La Cittá di Dio (pp. 65-67). Se añaden otra serie de artículos menores y poesías en maltés o italiano.

100.- FORAN, E.A., Augustinian Miscellanea. Illustred by-, edited by the Rev. with an Introd. by the Rev. T. F. RYAN s. j. London, Burns Oates \& Washbourne, 1931, VII-96 p. (Augustinian Miscellanea). Contiene amén de la Introducción, artículos de Foran, El congreso Eucarístico de Cartago, La Apertura del mismo, celebraciones del Centenario en Hipona y celebraciones y conferencias en Irlanda y en Westminster. Al final recoge la Enc. De Pío XI, Ad salutem humani generis, y antes dos artículos uno de Fr. Doolan y otro de J. Mahoney.

101.- Antonianum, vol. VI, fasc. ]: De S. Augustino. Romae, 1931, 112 pp. (=Antonianum).

Escriben B. Pergamo, J. Heerinckx, A. M. Vellici y F. Antonelli. Y entre las recensiones se halla De S. Augustini sermonibus en "Miscellanea Agostiniana".

102.- La celebrazione del XV Centenario della morte di S. Agostino nell' Abbazia di Rasazzo. Udine, Friulane, 1931, 28 pp.

103.- Settimana di Studi Agostiniani nell' Aula Magna dell'Universitá dal 27 aprile al 1mo maggio 1931. In occasione del XV Centenario del glorioso transito di S. Agostino, Vescovo e Dottor massimo della Chiesa 430-1930).Valletta, Tip. Giov. Muscat, 1932, 215 p.

Conferencias de D. Callus, Chetta Schivó Papás Francesco, E. Galea, P. Grech, V. Laurenza, A. Schembri, G. Spiteri, F. Puglisi, G. Monti.

104.- Augustinian Studies. Papers read at Recent Augustinian Educational Conferences. Washington, Saint Augustine's College, 1937, 193 pp. (= Augustinian Studies).

Artículos leídos en esas Conferencias. Se deben a la pluma de F. A. Tourscher, Kavanagh, Roland, Kehoe. Algunos no dicen relación con san Agustín, sino con la organización escolar americana para los agustinos. De poco valor científico y sí divulgativo.

JOSÉ MORÁN

Santibáñez de la Isla (León). 